\title{
Contour evolution scheme for variational image segmentation and smoothing
}

\author{
S. Mahmoodi and B.S. Sharif
}

\begin{abstract}
An algorithm, based on the Mumford-Shah $(\mathrm{M}-\mathrm{S})$ functional, for image contour segmentation and object smoothing in the presence of noise is proposed. However, in the proposed algorithm, contour length minimisation is not required and it is demonstrated that the $\mathrm{M}-\mathrm{S}$ functional without contour length minimisation becomes an edge detector. Optimisation of this nonlinear functional is based on the method of calculus of variations, which is implemented by using the level set method. Fourier and Legendre's series are also employed to improve the segmentation performance of the proposed algorithm. The segmentation results clearly demonstrate the effectiveness of the proposed approach for images with low signal-to-noise ratios.
\end{abstract}

\section{Introduction}

The problem of image segmentation and smoothing using variational methods has received considerable attention recently [1-5]. The snake segmentation algorithm based on methods of calculus of variations was first introduced by Kass et al. [1]. In another study, the level set method initially introduced in the area of fluid dynamics $[6,7]$ was proposed in [8] for image segmentation (see [9] for detailed discussions on the level set method). The snake algorithm proposed in [1] was further developed as a geodesic active contour model, which was based on the level set method with two different approaches in [10-12] and [13-15] by finding the geodesics of a feature space known to be a Riemannian manifold. In contrast, Mumford and Shah [2] introduced a nonlinear model for simultaneous segmentation and smoothing (also discussed in [16-18]), which was further approximated and implemented using various approaches ([3-5, 19-22]). This paper investigates the $\mathrm{M}-\mathrm{S}$ functional without the contour length minimisation term for image segmentation and smoothing, and its implementation is based on the Chan-Vese $(\mathrm{C}-\mathrm{V})$ model [4, 19-22]. Fourier and Legendre's series are also employed to enhance the performance of this region-based algorithm for different applications such as unsupervised texture segmentation. Initially, we briefly explain the $\mathrm{M}-\mathrm{S}$ functional in this section. For implementation purposes, the $\mathrm{C}-\mathrm{V}$ model $[4,19-22]$ is then introduced.

Image $g(x, y)$ is considered as a piecewise continuous function with contours $\Gamma$ representing discontinuities. $f(x, y)$ is the smoothed continuous function of class $C^{n} n \geq 2$ in $R-\Gamma$. The $\mathrm{M}-\mathrm{S}$ functional of the smoothed image $f$ and

(C) The Institution of Engineering and Technology 2007 doi:10.1049/iet-ipr:20050188

Paper first received 4th July 2005 and in final revised form 10th April 2007

S. Mahmoodi is with the Psychology Division, School of Psychology, Newcastle University, Henry Wellcome Building, Framlington Place, Newcastle upon Tyne NE2 4HH, UK

B.S. Sharif is with the School of Electrical, Electronic and Computer Engineering, Newcastle University, Merz Court, Newcastle upon Tyne, NE1 7RU, UK

E-mail: sasan.mahmoodi@ncl.ac.uk contour $\Gamma$ is then defined as [2]

$$
\begin{aligned}
E(f, \Gamma)= & \iint_{R}\left[(f(x, y)-g(x, y))^{2}\right] \mathrm{d} x \mathrm{~d} y \\
& +\mu \iint_{R-\Gamma}|\nabla f(x, y)|^{2} \mathrm{~d} x \mathrm{~d} y+\nu|\Gamma|
\end{aligned}
$$

where $R$ is a region in which $g(x, y)$ has no discontinuities, surrounded by discontinuity represented by $\Gamma$ whose length is $|\Gamma|, \mu$ and $\nu$ are non-negative constants and $\nabla$ denotes the gradient operator.

The Lagrange-Euler equations [23] with respect to the smoothed image $f$ and its derivatives for functional (1) can be written as a Helmholtz type differential equation [2] when contour $\Gamma$ is assumed fixed

$$
\mu \nabla^{2} f=f-g \quad \text { in } R-\Gamma
$$

with the Neumann boundary condition

$$
\frac{\partial f}{\partial n}=0 \quad \text { on } \Gamma
$$

where $\nabla^{2}$ is the Laplacian operator and $\partial / \partial n$ is the derivative along the normal direction to the contour.

The minimisation of the $\mathrm{M}-\mathrm{S}$ functional in a small neighbourhood of an arbitrary point $P$ on a discontinuity with respect to contour variations leads to the following nonlinear differential equation [2]

$$
\begin{aligned}
& \left(f^{+}(x, y)-g(x, y)\right)^{2}+\mu\left|\vec{\nabla} f^{+}\right|^{2}-\left(f^{-}(x, y)-g(x, y)\right)^{2} \\
& \quad-\mu\left|\vec{\nabla} f^{-}\right|^{2}+v \operatorname{Curv}\left(\Gamma_{P}\right)=0
\end{aligned}
$$

where $\Gamma_{P}$, Curv and $f^{+}$and $f^{-}$are the optimised curve in a small neighbourhood of $P$, an operator representing curvature and the variations of the smoothed function $f$ because of contour variation in two opposite directions, respectively. It should be noted that in the $\mathrm{M}-\mathrm{S}$ functional, $f$, which is a twodimensional manifold, belongs to an appropriate Banach space, whereas $\Gamma$, which is a one-dimensional manifold, is not associated with any known space.

The $\mathrm{C}-\mathrm{V}$ model proposed in $[4,19-22]$ to implement the $\mathrm{M}-\mathrm{S}$ functional is a level-set-based method that uses a 
Lipschitz function $\varphi(x, y)$, whose front represents an evolving contour $[8,9]$, to separate the whole image region into two inside and outside sub-regions. This function is initialised as a signed distance function [4]. In the piecewise constant approximation of the $\mathrm{M}-\mathrm{S}$ functional, the $\mathrm{C}-\mathrm{V}$ functional is written as [4]

$$
\begin{aligned}
F\left(k_{\mathrm{i}}, k_{\mathrm{o}}, \varphi\right)= & \iint_{R}\left(g(x, y)-k_{\mathrm{i}}\right)^{2} H_{\varepsilon}(\varphi) \mathrm{d} x \mathrm{~d} y \\
& +\iint_{R}\left(g(x, y)-k_{\mathrm{o}}\right)^{2}\left(1-H_{\varepsilon}(\varphi)\right) \mathrm{d} x \mathrm{~d} y \\
& +v \iint_{R} \delta_{\varepsilon}(\varphi)|\nabla \varphi| \mathrm{d} x \mathrm{~d} y
\end{aligned}
$$

where $H_{\varepsilon}$ and $\delta_{\varepsilon}$ are the regularised Heaviside (step) function and the Dirac delta function, respectively. $H_{\varepsilon}$ is regularised as

$$
H_{\varepsilon}(z)=\frac{1}{2}\left(1+\frac{2}{\pi} \arctan \left(\frac{z}{\varepsilon}\right)\right)
$$

where $\varepsilon=1$ in this paper, and $k_{\mathrm{i}}$ and $k_{\mathrm{o}}$ are the mean values of the image inside and outside of the evolving contour, respectively. Minimisation of functional (5) with respect to $k_{\mathrm{i}}$ and $k_{\mathrm{o}}$ and leads to

$$
k_{\mathrm{i}}(\varphi)=\frac{\iint_{R} g(x, y) H_{\varepsilon}(\varphi(x, y)) \mathrm{d} x \mathrm{~d} y}{\iint_{R} H_{\varepsilon}(\varphi(x, y)) \mathrm{d} x \mathrm{~d} y}
$$

and

$$
k_{\mathrm{o}}(\varphi)=\frac{\iint_{R} g(x, y)\left(1-H_{\varepsilon}(\varphi(x, y))\right) \mathrm{d} x \mathrm{~d} y}{\iint_{R}\left(1-H_{\varepsilon}(\varphi(x, y))\right) \mathrm{d} x \mathrm{~d} y}
$$

According to [4], the minimisation of functional (5) with respect to $\varphi(x, y)$ leads to the following Euler-Lagrange equation

$$
\frac{\partial \varphi}{\partial t}=\delta_{\varepsilon}(\varphi)\left[\nu \nabla \cdot\left(\frac{\nabla \varphi}{|\nabla \varphi|}\right)-\left(g(x, y)-k_{\mathrm{i}}\right)^{2}+\left(g(x, y)-k_{\mathrm{o}}\right)^{2}\right]
$$

Functional (5), in piecewise continuous approximation of (1), is modified to

$$
\begin{aligned}
F\left(f_{\mathrm{i}}, f_{\mathrm{o}}, \varphi\right) & =\iint_{R}\left\lfloor\left(g(x, y)-f_{\mathrm{i}}\right)^{2}+\mu\left|\nabla f_{\mathrm{i}}\right|^{2}\right\rfloor H_{\varepsilon}(\varphi) \mathrm{d} x \mathrm{~d} y \\
& +\iint_{R}\left\lfloor\left(g(x, y)-f_{\mathrm{o}}\right)^{2}+\mu\left|\nabla f_{\mathrm{o}}\right|^{2}\right\rfloor\left(1-H_{\varepsilon}(\varphi)\right) \mathrm{d} x \mathrm{~d} y \\
& +v \iint_{R} \delta_{\varepsilon}(\varphi)|\nabla \varphi| \mathrm{d} x \mathrm{~d} y
\end{aligned}
$$

where $f_{\mathrm{i}}$ and $f_{\mathrm{o}}$ are the smoothed images inside and outside the evolving contour and are calculated as [19]

$$
\begin{array}{lll}
\mu \nabla^{2} f_{\mathrm{i}}=f_{\mathrm{i}}-g & \text { for } & \varphi(x, y)>0 \\
\mu \nabla^{2} f_{\mathrm{o}}=f_{\mathrm{o}}-g & \text { for } & \varphi(x, y)<0
\end{array}
$$

Minimisation of functional (9) with respect to $\varphi(x, y)$ results in

$$
\begin{aligned}
\frac{\partial \varphi}{\partial t}= & \delta_{\varepsilon}(\varphi)\left[\nu \nabla \cdot\left(\frac{\nabla \varphi}{|\nabla \varphi|}\right)-\left(g(x, y)-f_{\mathrm{i}}\right)^{2}-\mu\left|\nabla f_{\mathrm{i}}\right|^{2}\right. \\
& \left.+\left(g(x, y)-f_{\mathrm{o}}\right)^{2}+\mu\left|\nabla f_{\mathrm{o}}\right|^{2}\right]
\end{aligned}
$$

Piecewise constant and continuous approximations of the M-S model proposed by Chan and Vese [4, 19-22] and presented in (6) and (8) (for piecewise constant) and (10)-(12) (for piecewise continuous) can be implemented iteratively, where for each iteration, $\varphi(x, y)$ is calculated using (8) or (12). In each iteration, either (6) and (7) or (10) and (11) are then employed to calculate the mean values or the smoothed functions inside and outside the evolving contour.

In this paper, three modifications to the $\mathrm{M}-\mathrm{S}$ and $\mathrm{C}-\mathrm{V}$ models are proposed: (i) contour length minimisation term is dropped; (ii) Gaussian filter is applied to $\varphi(x, y)$ in every iteration to improve the performance of the algorithm in very noisy images and (iii) $\mathrm{C}-\mathrm{V}$ models proposed in [4, $19,22]$ are extended to piecewise polynomials and Fourier series. The derivations and implementations of these modifications are presented in Section 2. Results are discussed in Section 3, and conclusions are drawn in Section 4.

\section{Modifications and implementation}

In this work, to implement the modifications described in the previous section, the framework employed in the $\mathrm{C}-\mathrm{V}$ model is used, and a Lipschitz function $\varphi[8,9]$ is used in this framework. The zero level of this function known as 'front' represents the contours $\Gamma$ 's of the objects in an image. Function $\varphi$ is therefore initialised as the signed distance function between every point of the domain and the initial contour, such that $\varphi(x, y)=0, \varphi(x, y)>0$ and $\varphi(x, y)<0$ correspond to the contour and the regions inside and outside the contour, respectively $[4,8,9,19-$ 22]. Therefore as the contour evolves through the segmentation process, the algorithm always follows $\varphi(x, y)=0$, representing the evolving contour.

The first modification is proposed to remove the requirement of contour length minimisation. Our rationale is that contours representing discontinuities are minimisers of functional (1) when $v=0$. Such a functional can be written as

$$
\begin{aligned}
E(f, \Gamma)= & \iint_{R}\left[(f(x, y)-g(x, y))^{2}\right] \mathrm{d} x \mathrm{~d} y \\
& +\mu \iint_{R-\Gamma}|\nabla f(x, y)|^{2} \mathrm{~d} x \mathrm{~d} y
\end{aligned}
$$

The proposed modification is not trivial, because it involves ensuring the second variation of functional (13) to be positive with respect to contour variations in order to discard unwanted contours. Functional (13) was initially developed for one-dimensional signals [24, 25], and it was demonstrated in [25] that its second variation with respect to points representing discontinuities is positive. This indicates that points representing discontinuities are minimisers of such a functional. A method based on second variation is also employed to detect discontinuity points in a signal represented by piecewise continuous functions. The same notion is applicable to contours representing discontinuities in images, and therefore in this paper, the contour length minimisation term in functional (1) is dropped. To 
implement the functional, we employ the $\mathrm{C}-\mathrm{V}$ framework as described in the previous section to write the functional in piecewise constant approximation as

$$
\begin{aligned}
F\left(k_{\mathrm{i}}, k_{\mathrm{o}}, \varphi\right) & =\iint_{R}\left(g(x, y)-k_{\mathrm{i}}\right)^{2} H_{\varepsilon}(\varphi(x, y)) \mathrm{d} x \mathrm{~d} y \\
& +\iint_{R}\left(g(x, y)-k_{\mathrm{o}}(x, y)\right)^{2}\left(1-H_{\varepsilon}(\varphi(x, y))\right) \mathrm{d} x \mathrm{~d} y
\end{aligned}
$$

The minimisation of functional (14) with respect to $\varphi$ is written as

$$
\frac{\partial \varphi}{\partial t}=\delta_{\varepsilon}(\varphi)\left[-\left(g-k_{\mathrm{i}}\right)^{2}+\left(g-k_{\mathrm{o}}\right)^{2}\right]
$$

where $H_{\varepsilon}$ and $\delta_{\varepsilon}$ are the regularised Heaviside (step) and the Dirac delta functions, respectively, and $k_{\mathrm{i}}$ and $k_{\mathrm{o}}$ are calculated using (6) and (7). Equation (15) converges when the evolving contour corresponds to discontinuity. The positivity requirement for the second variation of functional (14) can be simply met by examining $k_{\mathrm{i}}$ and $k_{\mathrm{o}}$. If in convergence, $k_{\mathrm{i}}=k_{\mathrm{o}}$, then the contour does not correspond to any discontinuity and should be discarded. If there is only one type of foreground and one type of background, then there is no need to examine $k_{\mathrm{i}}$ and $k_{\mathrm{o}}$, and the contour automatically converges to discontinuities.

It is well known that the piecewise continuous approximation of the $\mathrm{C}-\mathrm{V}$ model (functional 9) falls into local minima [19] particularly when the initial contour is not close to any discontinuity. It is also numerically expensive to calculate the smoothed images and the Lipschitz function $\varphi$ by solving the three partial differential equations (10)(12) in each iteration. Therefore we propose our second modification for the piecewise continuous approximation to address the above two issues.

In order to find the global minimum in such cases, the smoothed image $f(x, y)$ is approximated by using a weighted sum of a series of eigenfunctions such as Legendre and/or Fourier series whose coefficients are calculated iteratively. As the eigenfunctions are continuous functions, their weighted sum is also a continuous function.

Legendre polynomials [26] can be used to parameterise image fluctuations using a set of coefficients, where the desired solution $f(x, y)$ inside and outside the contour is initially approximated as the weighted sum of Legendre polynomials. This approximation up to the first order $(n=1)$ was initially proposed in [22]. Here, we develop this approximation method further. It is well known that Legendre polynomials are orthogonal and hence can be considered as a set of eigenfunctions. Orthogonal property for a Legendre polynomial $P_{n}(x)$ of degree $n$ can be written as

$$
\int_{-1}^{+1} P_{m}(x) P_{n}(x) \mathrm{d} x=\frac{2}{2 n+1} \delta_{m n}
$$

where $\delta_{m n}$ is the Kronecker delta.

Therefore any $C^{n}$ function with $n \geq 1$ can be described as a series of Legendre polynomials, that is, $f(x, y)$ can be approximated as

$$
f(x, y)=\sum_{n} \sum_{m} k_{m n} P_{n}(x) P_{m}(y)
$$

where $k_{m n}$ are Legendre coefficients associated with $f(x, y)$.
In the level set framework used in this paper, an approximation of the image inside the contour can be represented as

$$
f_{\mathrm{i}}(x, y)=\sum_{n} \sum_{m} k_{i m n} P_{n}(x) P_{m}(y)
$$

where $k_{i m n}$ are the coefficients that describe the image inside the contours.

Similarly, for a region of the image outside the contour, we have

$$
f_{\mathrm{o}}(x, y)=\sum_{n} \sum_{m} k_{\text {omn }} P_{n}(x) P_{m}(y)
$$

Functional (13) can therefore be rewritten as

$$
\begin{aligned}
F\left(\boldsymbol{k}_{\mathrm{i}}, \boldsymbol{k}_{\mathrm{o}}, \varphi\right)= & \iint_{R}\left(g(x, y)-\sum_{n} \sum_{m} k_{i m n} P_{n}(x) P_{m}(y)\right)^{2} \\
& \times H(\varphi(x, y)) \mathrm{d} x \mathrm{~d} y \\
& +\iint_{R}\left(g(x, y)-\sum_{n} \sum_{m} k_{\text {omn }} P_{n}(x) P_{m}(y)\right)^{2} \\
& \times(1-H(\varphi(x, y))) \mathrm{d} x \mathrm{~d} y
\end{aligned}
$$

where $\boldsymbol{k}_{\mathrm{i}}$ and $\boldsymbol{k}_{\mathrm{o}}$ are the vector parameters defined as

$$
\boldsymbol{k}_{\mathrm{i}}=\left(\boldsymbol{k}_{\mathrm{i} 00}, \boldsymbol{k}_{\mathrm{i} 01}, \boldsymbol{k}_{\mathrm{i} 10}, \ldots\right) \text { and } \boldsymbol{k}_{\mathrm{o}}=\left(\boldsymbol{k}_{\mathrm{o} 00}, \boldsymbol{k}_{\mathrm{o} 01}, \boldsymbol{k}_{\mathrm{o} 10}, \ldots\right)
$$

Functional (19) should be minimised with respect to $\boldsymbol{k}_{\mathrm{i}}$ and $\boldsymbol{k}_{\mathrm{o}}$, and, which leads to a linear system with $N$ equations and $N$ unknowns, where $N$ is the number of terms used in the Legendre series

$$
\begin{aligned}
& \iint_{R} P_{p}(x) P_{q}(y) g(x, y) H(\varphi(x, y)) \mathrm{d} x \mathrm{~d} y \\
& \quad=\sum_{n} \sum_{m} k_{i m n} \iint_{R} P_{p}(x) P_{q}(y) P_{n}(x) P_{m}(y) H(\varphi(x, y)) \mathrm{d} x \mathrm{~d} y
\end{aligned}
$$

where $p, q \geq 0$.

A similar linear system to (20) can be derived for $\boldsymbol{k}_{\mathrm{o}}$. By optimising functional (19) with respect to $\varphi(x, y)$ using the Euler-Lagrange equation, differential equation (21) is obtained

$$
\begin{aligned}
\frac{\partial \varphi}{\partial t}= & \delta_{\varepsilon}(\varphi)\left[-\left(g(x, y)-\sum_{n} \sum_{m} k_{i m n} P_{n}(x) P_{m}(y)\right)^{2}\right. \\
& \left.+\left(g(x, y)-\sum_{n} \sum_{m} k_{\text {omn }} P_{n}(x) P_{m}(y)\right)^{2}\right]
\end{aligned}
$$

Differential equation (21) coupled with linear system (20) is the optimal solution of functional (19) and can be numerically implemented to find the global minimum. The shifted Legendre polynomials are employed in the implementation, as it is easier to consider the image domain as $[0,1] \times[0,1]$.

Another approach to parameterise an $M \times N$ image using eigenfunctions is to use Fourier series to approximate the desired function $f(x, y)(M \times N)$ using half-range expansion [26], that is

$$
f(x, y)=\sum_{m} \sum_{n} k_{m n} \cos (m u x+n v y)
$$

where $u=2 \pi / M, v=2 \pi / N$, and $k_{m n}$ are spatial frequencies and Fourier coefficients, respectively. Functional (13) 


$$
\begin{aligned}
F\left(\boldsymbol{k}_{\mathrm{i}}, \boldsymbol{k}_{\mathrm{o}}, \varphi\right)= & \iint_{R}\left(g(x, y)-\sum_{m} \sum_{n} k_{i m n} \cos (m u x+n v y)\right)^{2} \\
& \times H(\varphi(x, y)) \mathrm{d} x \mathrm{~d} y+\iint_{R}(g(x, y) \\
& \left.-\sum_{m} \sum_{n} k_{o m n} \cos (m u x+n v y)\right)^{2} \\
& \times(1-H(\varphi(x, y))) \mathrm{d} x \mathrm{~d} y
\end{aligned}
$$

Functional (22) should be minimised with respect to $k_{i p q}$ and $k_{o p q}$. The following linear system is obtained by optimising functional (22) with respect to $k_{i p q}$

$$
\begin{aligned}
& \iint_{R} \cos (p u x+q v y) g(x, y) H(\varphi(x, y)) \mathrm{d} x \mathrm{~d} y \\
& =\sum_{m} \sum_{n} k_{m n} \iint_{R} \cos (p u x+q v y) \cos (m u x+n v y) \\
& \quad \times H(\varphi(x, y)) \mathrm{d} x \mathrm{~d} y, \quad p, q \geq 0
\end{aligned}
$$

A similar linear system can be derived for $k_{o p q}$ coefficients. The following differential equation can be further derived by applying the Euler-Lagrange equation on functional (22) to optimise the functional with respect to $\varphi(x, y)$

$$
\begin{aligned}
\frac{\partial \varphi}{\partial t}= & \delta_{\varepsilon}(\varphi)\left[-\left(g-\sum_{m} \sum_{n} k_{\text {imn }} \cos (m u x+n v y)\right)^{2}\right. \\
& \left.+\left(g-\sum_{m} \sum_{n} k_{\text {omn }} \cos (m u x+n v y)\right)^{2}\right]
\end{aligned}
$$

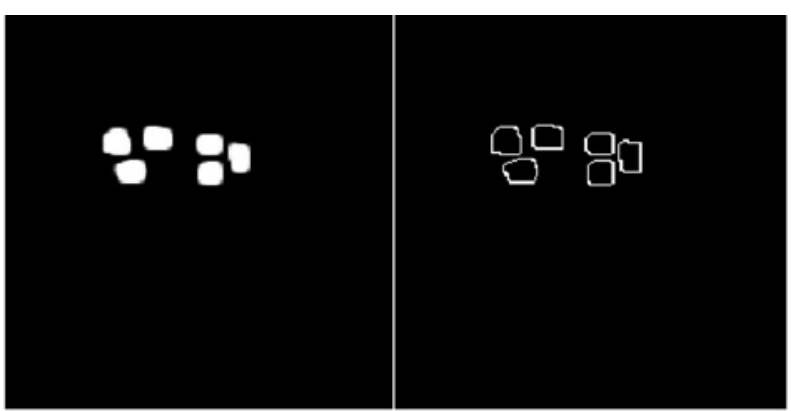

a

b

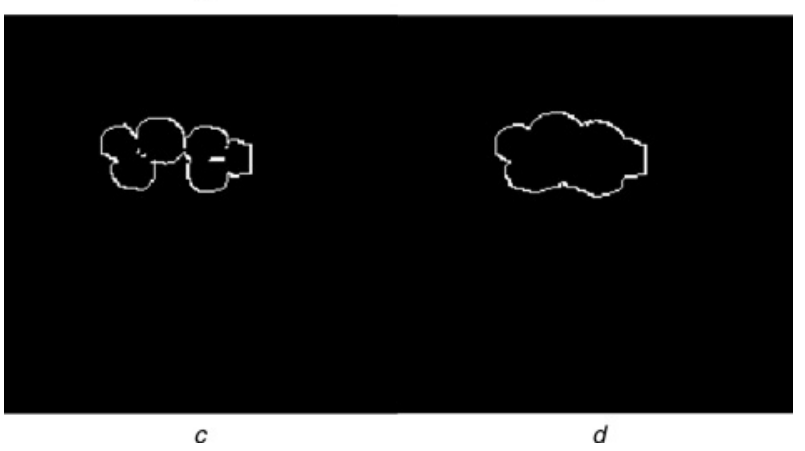

Fig. 1 Image containing a cluster of objects and its segmented images with different values for $v$

$a$ Original image containing a cluster of objects

$b$ Segmented image using piecewise constant approximation without contour length minimisation

c Segmented image using piecewise constant approximation with contour length minimisation $v=50$

$d$ Segmented image using piecewise constant approximation with contour length minimisation $v=70$
Differential equation (24) coupled with linear system (23) can then be implemented to iteratively solve the optimisation problem in functional (22). This leads to a set of coefficients $k_{\text {imn }}$ and $k_{\text {omn }}$ that describe $f_{\mathrm{i}}(x, y)$ and $f_{\mathrm{o}}(x, y)$ and a function $\varphi(x, y)$ whose zero level describes the contour. It is noted that (21) and (24) converge in discontinuities. The positivity requirement of the second variation of functionals (19) and (22) can be met by examining vectors $\boldsymbol{k}_{\mathrm{i}}$, and $\boldsymbol{k}_{\mathrm{o}}$. If under convergence $\boldsymbol{k}_{\mathrm{i}}=\boldsymbol{k}_{\mathrm{o}}$, then the contour should be discarded. However, for images with only one type of foreground and one type of background, the algorithm automatically converges to discontinuities without examining vectors $\boldsymbol{k}_{\mathrm{i}}$ and $\boldsymbol{k}_{\mathrm{o}}$.

With excessive amount of noise, the problem of segmentation discussed in this section becomes ill-posed. To regularise the problem, we propose another modification to apply a twodimensional Gaussian low-pass filter to the function $\varphi(x, y)$ at every iteration. As will be observed, this causes the snake algorithm to operate successfully with images characterised with signals-to-noise ratios (SNRs) as low as 0.1. Upon convergence, reconstruction can be performed using parameters calculated during the segmentation process. The reconstructed image, which can be viewed as the smoothed image, is therefore viewed as a by-product of the proposed segmentation process. To summarise, the proposed algorithm consists of the following steps.

1. Initialise $\varphi^{0}$ for $n=0$.

2. Compute $\boldsymbol{k}_{\mathrm{i}}$ and $\boldsymbol{k}_{\mathrm{o}}$.

3. Solve differential equation for $\varphi$ to obtain $\varphi^{n+1}$.

4. Apply Gaussian low-pass filter to $\varphi^{n+1}$.

5. Check if convergence is reached. If not, $n=n+1$ and go to step 2; otherwise go to step 6.

6. Reconstruct the image using $\boldsymbol{k}_{\mathrm{i}}$ and $\boldsymbol{k}_{\mathrm{o}}$.

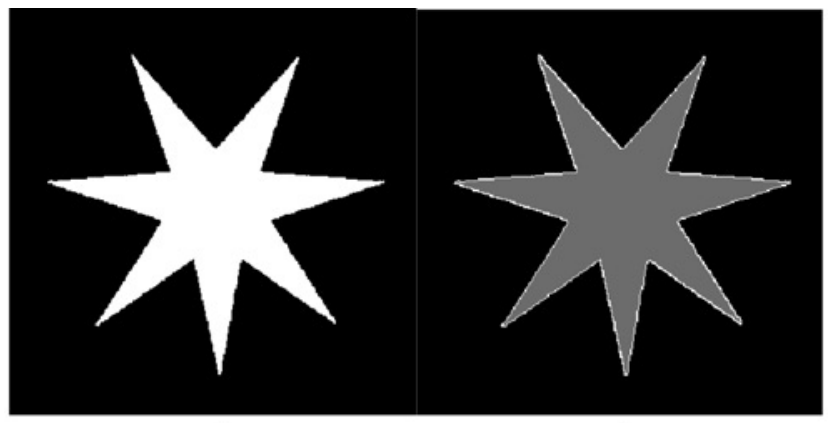

$b$

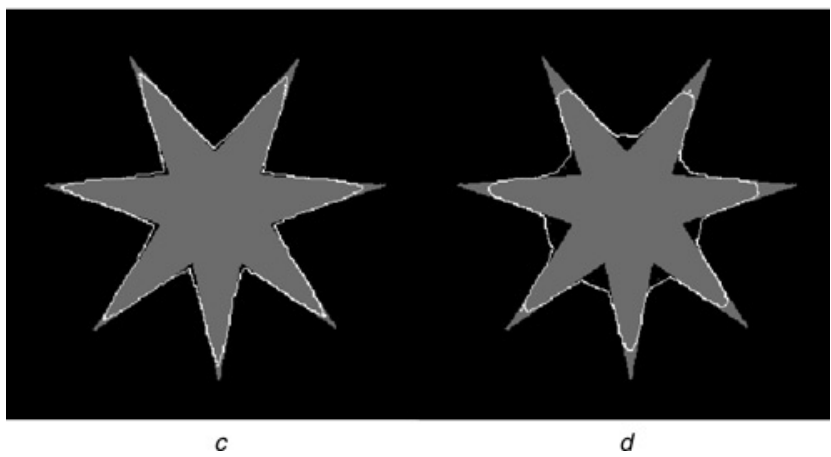

Fig. 2 Synthetic star image and its segmented images with different values for $v$

$a$ Original star image

$b$ Segmented image using piecewise constant approximation without contour length minimisation

$c$ Segmented image using piecewise constant approximation with contour length minimisation $v=300$

$d$ Segmented image using piecewise constant approximation with contour length minimisation $v=600$ 


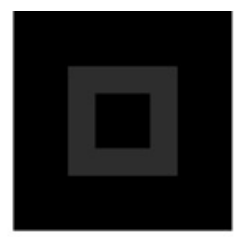

a

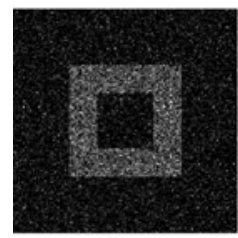

$b$

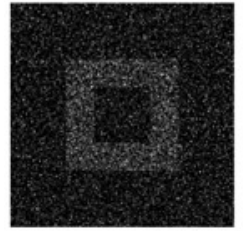

e

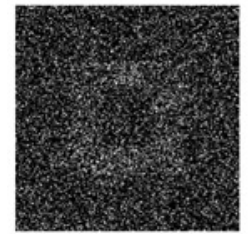

h
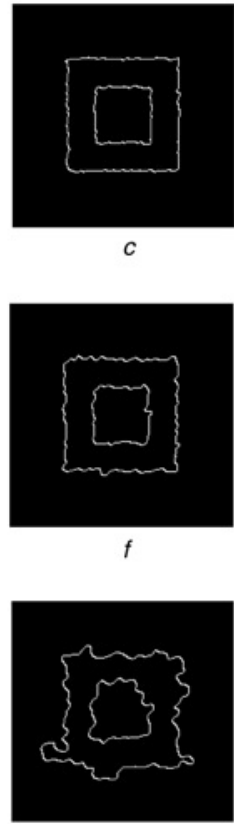

i

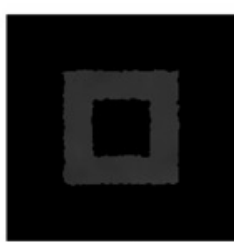

$d$

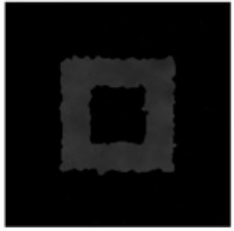

$g$

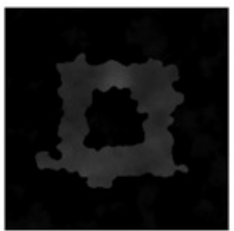

Fig. 3 Noise sensitivity of the proposed algorithm

$a$ Original noiseless image

$b$ Image contaminated with Gaussian noise with $\mathrm{SNR}=1.088$

c Segmentation results for $\mathrm{SNR}=1.088$

$d$ Smoothed image with $\mu=100$ for $\mathrm{SNR}=1.088$

$e$ Image contaminated with Gaussian noise with $\mathrm{SNR}=0.372$

$f$ Segmentation results for $\mathrm{SNR}=0.372$

$g$ Smoothed image with $\mu=100$ for $\mathrm{SNR}=0.372$

$h$ Image contaminated with Gaussian noise with SNR $=0.051$

$i$ Segmentation results for $\mathrm{SNR}=0.051$

$j$ Smoothed image with $\mu=100$ for $\mathrm{SNR}=0.051$

In the next section, the results achieved by the above algorithms are presented.

\section{Results}

In this section, the algorithms outlined in the previous sections are applied to different synthetic and real world images. In Figs. 1 and 2, we specifically address the impact of removing the contour length minimisation term in the $\mathrm{M}-\mathrm{S}$ functional. Equations (6)-(8) (piecewise constant approximation with contour length minimisation term) are applied to the image shown in Fig. 1a with $v=50$ and $v=70$. The detected contours are shown in Figs. $1 c$ and $1 d$. Equations (6), (7) and (15) (piecewise constant approximation without contour length minimisation term) are also applied to the image of Fig. 1 $a$. The segmented image is depicted in Fig. 1b. It is clear from Fig. 1 that when the contour length minimisation term is included in the $\mathrm{M}-\mathrm{S}$ functional, the contours detected do not correspond with the edges of the object in a given image. This is expected in the $\mathrm{M}-\mathrm{S}$ functional, as the contour minimisation term smoothes the detected contour. The extent of the difference between the contours and the objects' actual edges in a given image depends on the value chosen for $v$ and the image contents, i.e. $k_{\mathrm{i}}$ and $k_{\mathrm{o}}$ in equation (8).

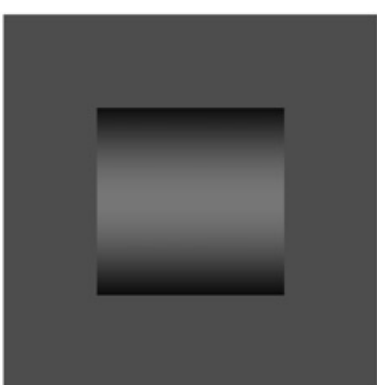

a

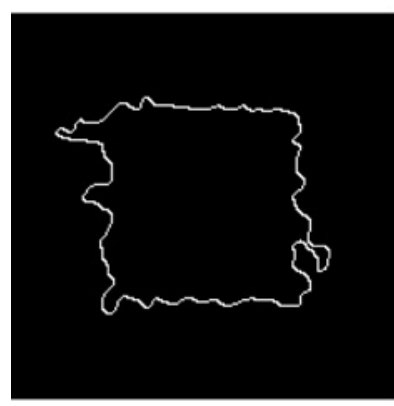

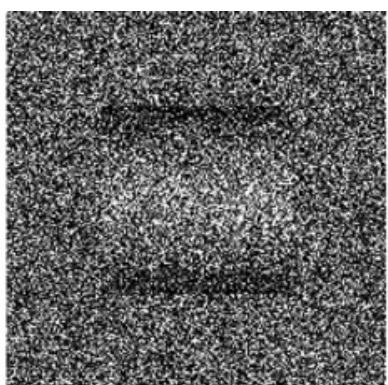

$b$

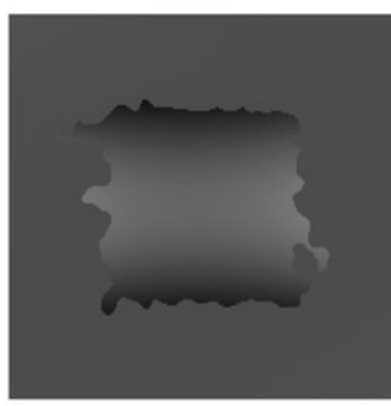

$d$
Fig. 4 Approximation of the functional using Legendre series $a$ Original noiseless image with a patch characterised by smooth variation in brightness

$b$ Noisy image with Gaussian noise $(\mathrm{SNR}=1.026)$

c Segmentation result using proposed algorithm based on Legendre series with the first six Legendre components

$d$ Reconstructed image using the coefficients of the first six Legendre components

Fig. 2 shows another example that demonstrates the fact that in the $\mathrm{M}-\mathrm{S}$ functional, the detected contours do not always correspond with objects' edges. Equations (6)-(8) (piecewise constant approximation with contour length minimisation term) with $v=300$ and $v=500$ are applied

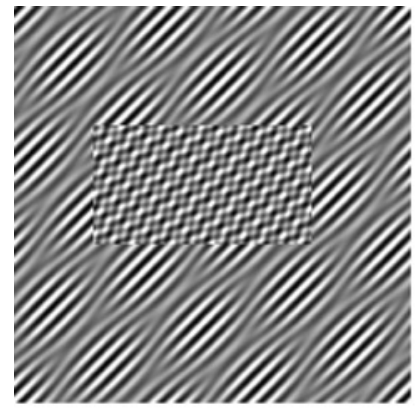

a

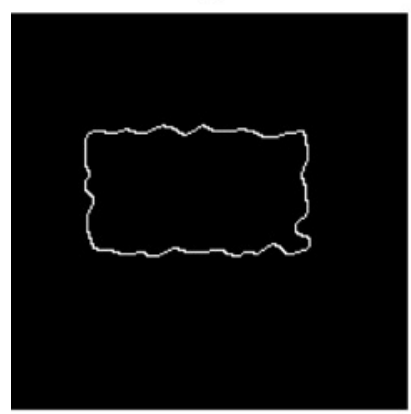

c

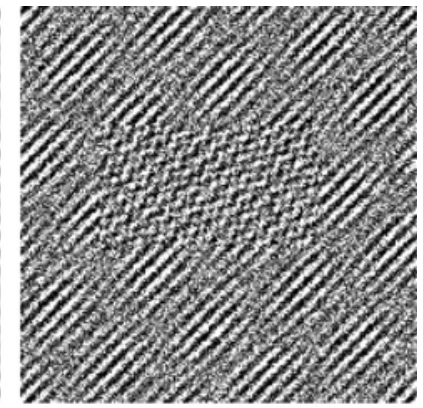

b

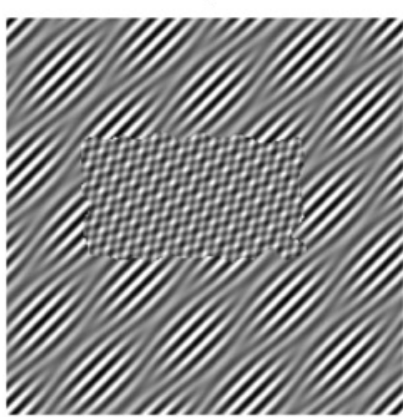

d
Fig. 5 Noiseless synthetic image

$a$ Original noiseless image with two different textures

$b$ Noisy image with Gaussian noise $(\mathrm{SNR}=7.347)$

$c$ Segmented image using our Fourier-based algorithm using 45 Fourier components

$d$ Reconstructed image using the coefficients of Fourier series 

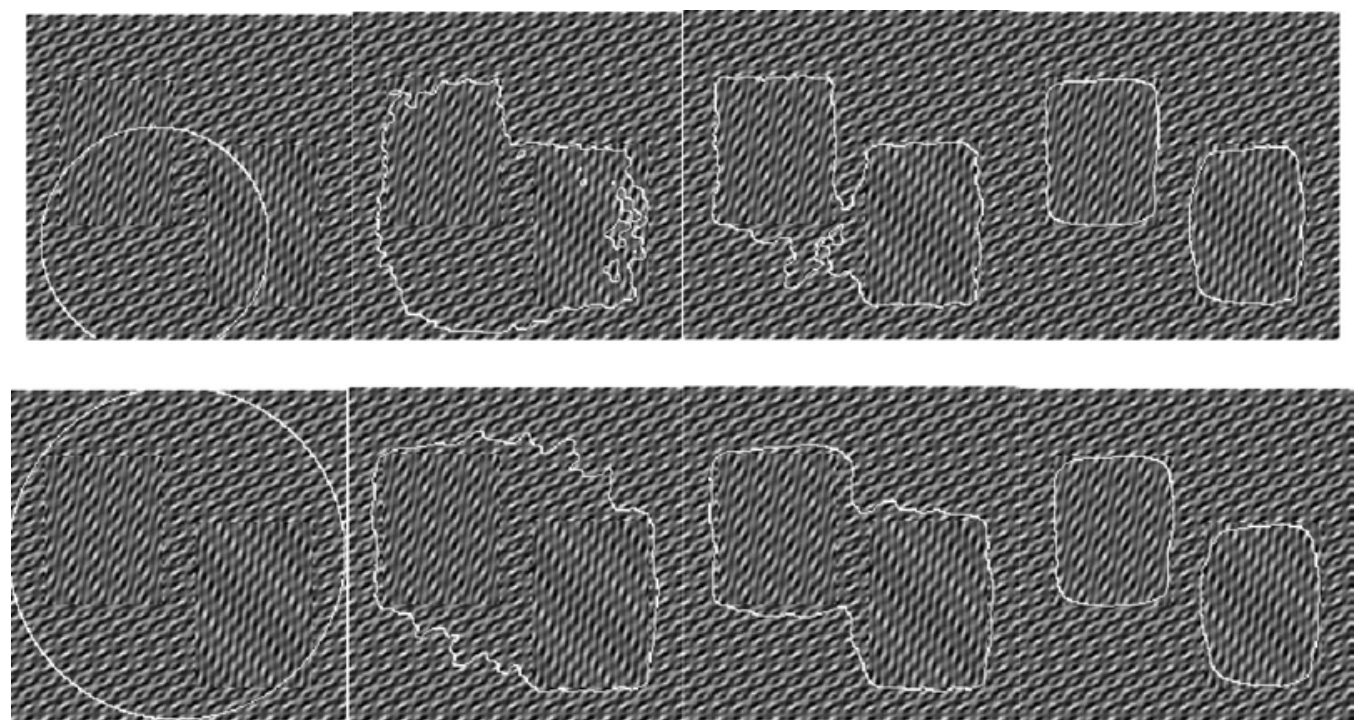

Fig. 6 Contour evolutions of the proposed Fourier-series-based method with two different initial contours (conditions)

to Fig. $2 a$ and the detected contours are depicted in Figs. $2 c$ and $2 d$. Fig. $2 b$ also shows the detected contour without the contour length minimisation term using piecewise constant approximation. For illustration and comparison purposes, the detected contours are superimposed on the original image in this figure. Figs. 1 and 2 therefore demonstrate that the $\mathrm{M}-\mathrm{S}$ functional without the contour minimisation term reduces to an edge detection scheme.

Noise sensitivity of the proposed algorithm is investigated in Fig. 3.

A noiseless image is considered in Fig. $3 a$, and Gaussian noise is added ( $\mathrm{SNR}=1.088)$ to obtain the image of Fig. $3 b$. The segmented image is shown in Fig. $3 c$ with a Gaussian filter bandwidth of $\pi / 3$, and the smoothed image depicted in Fig. $3 d$ is obtained with $\mu=100$. Similar results are shown in Fig. 3 for the same image with $\mathrm{SNR}=0.372$ and 0.051 with bandwidths being $\pi / 4$ and $\pi / 5$ for the Gaussian filter, respectively. As observed from Figs. $3 h-j$, the algorithm operates at SNR as low as 0.051 .

Approximation of the desired function $f(x, y)$ using Legendre series is demonstrated in Fig. $4 a$, which shows a patch characterised by a smooth variation in brightness against a background with a mean grey scale equal to that of the patch. This image is further contaminated with Gaussian noise (SNR =1.026) to obtain Fig. 4b. Coupled equations (20) and (21) are applied to the image of Fig. $4 b$ to achieve the segmented image shown in Fig. $4 c$ using the first six components in the Legendre series. The bandwidth of the Gaussian filter was chosen as $\pi / 6$. It should be noted that the both the piecewise constant (equations (6)-(8)) and continuous (equations (10)-(12)) solutions presented in $[4,19,21]$ fail to segment the patch. In the case of the piecewise constant solution, this is because the mean grey level of the central object in Fig. 4 is equal to the mean grey level of the rest of the image. Hence, there is no force to drive the evolving contour in (8). However, for piecewise continuous approximation, if the initial contour is not close enough to the object to be segmented, the $\mathrm{C}-\mathrm{V}$ method falls into local minima [19]. The reconstructed image formed by using the calculated coefficients of Legendre series at the convergence of the algorithm is depicted in Fig. $4 d$.

A noiseless synthetic image with two different textures is depicted in Fig. 5a. This image is contaminated with Gaussian noise $(\mathrm{SNR}=7.347)$ and shown in Fig. $5 b$. The segmentation result using the algorithm expressed in (23) and (24) is shown in Fig. 5c. The reconstructed image using the calculated coefficients of the Fourier series is depicted in Fig. $5 d$.

Fig. 6 depicts a few iterations of the contour evolutions of the proposed Fourier-based method in this paper, with two different initial contours applied to a synthetic image with two rectangular patches with the same texture against a different background texture.

The same image shown in Fig. 6 is also used in Fig. 7. Zero-mean Gaussian noise is added $(\mathrm{SNR}=1.061)$ to the

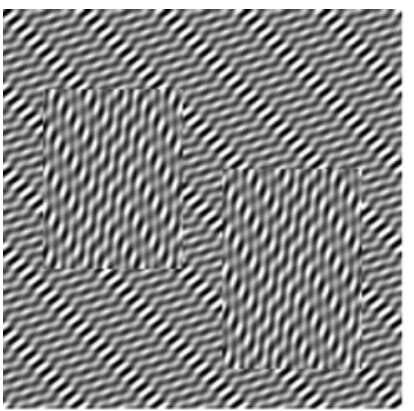

a

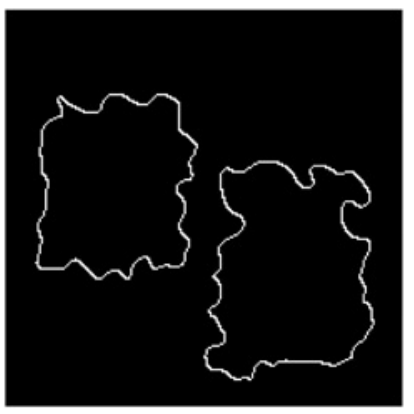

c

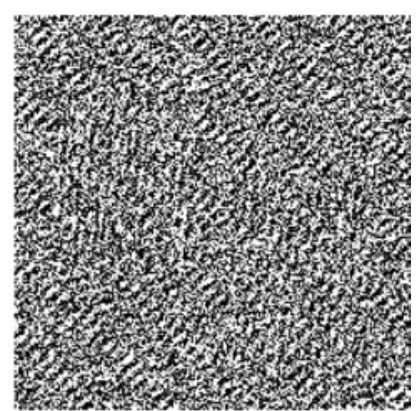

$b$

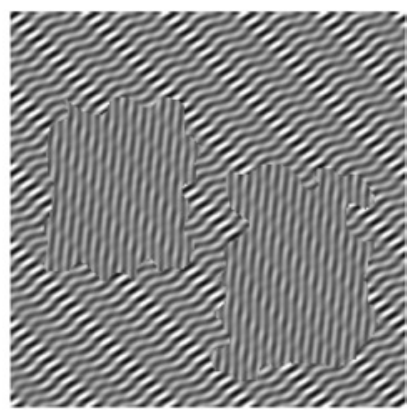

d
Fig. 7 Synthetic noisy textured image and its segmentation and reconstruction results

$a$ Original noiseless image with two separate regions with same texture against a background texture different from those regions $b$ Noisy image with additive zero-mean Gaussian noise $(\mathrm{SNR}=1.061)$

$c$ Segmented image using proposed Fourier-based algorithm using 55 Fourier components

$d$ Reconstructed image using the coefficients of Fourier series 


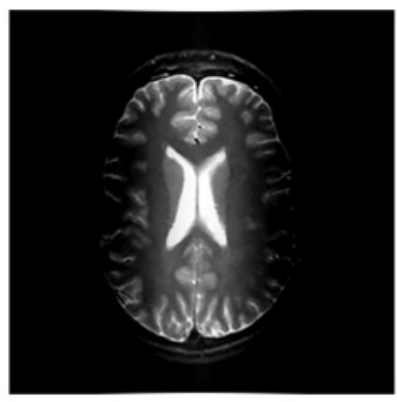

$a$

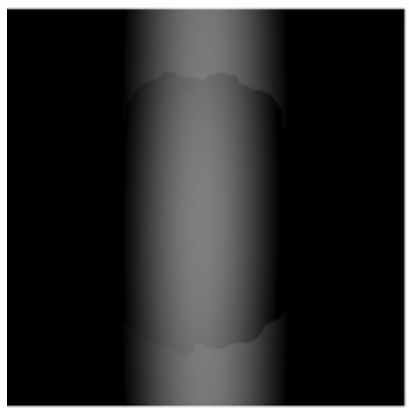

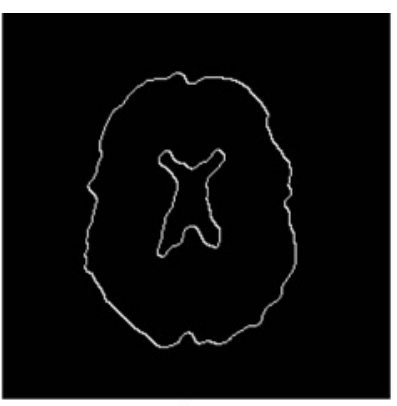

$b$

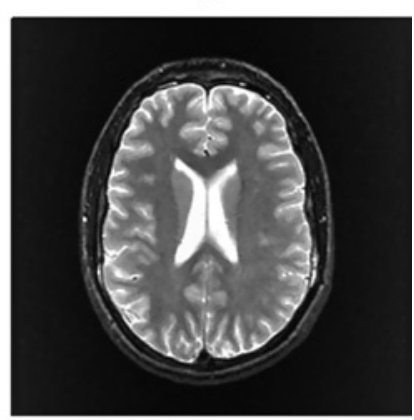

d
Fig. 8 Removal of distortion

$a$ Disorted MRI image

$b$ Segmentation using the algorithm based on the proposed Legendre series using the first six Legendre components

$c$ Distorting image estimated using the coefficients of Legendre series calculated in the process of segmentation

$d$ Corrected image after removing the distorting image

noiseless image of Fig. $7 a$ to obtain the noisy image shown in Fig. $7 b$. The algorithm described by (23) and (24) is applied to the noisy image, and the result is shown in Fig. $7 c$. Fig. $7 d$ shows the reconstructed image using the coefficients of the Fourier series. Careful inspection of the noisy image in Fig. $7 b$ demonstrates the challenge presented to human perception to recognise the presence of the objects in the image.

An important feature of the segmentation algorithm based on the Legendre series is the removal of distortion, which is demonstrated in Fig. 8. A magnetic resonance image distorted in the process of formation and digitisation is considered, as shown in Fig. $8 a$. The proposed algorithm based on the Legendre series is applied to the image, and the segmentation result is depicted in Fig. 8b. The distorting component in the image is estimated using the coefficients of the Legendre series calculated in the process of segmentation

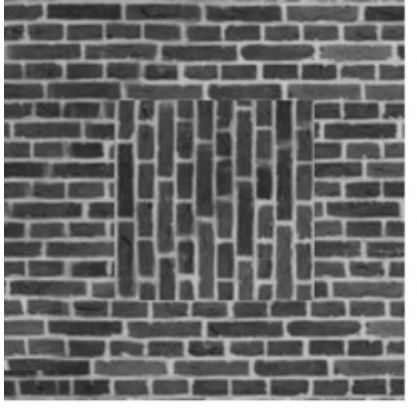

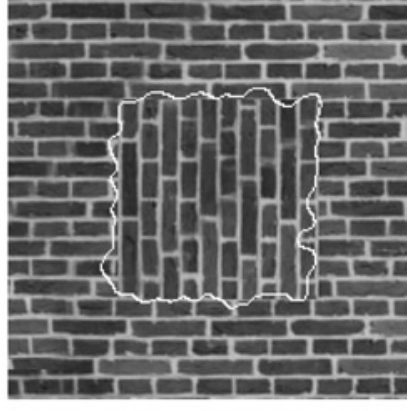

$b$
Fig. 9 Image with different wall patterns and segmentation result

$a$ Image with two different wall patterns

$b$ Segmentation result using 55 Fourier components superimposed on the original image and is shown in Fig. $8 c$. Fig. $8 d$ shows the corrected image after distortion removal from the original image.

Finally, the Fourier-based algorithm proposed in this paper is applied to an image with two different wall patterns, as shown in Fig. 9a. The segmentation result using 55 Fourier components is depicted in Fig. $9 b$.

\section{Conclusion}

An image segmentation and smoothing method based on the $\mathrm{M}-\mathrm{S}$ functional has been investigated in this paper. In this method, there is no requirement for contour length minimisation. Therefore the detected contours correspond to edges (discontinuities) in a given image. A two-dimensional low-pass filter is also added to improve the performance of the algorithm in a noisy environment. New methods based on Legendre and Fourier's series are proposed to find the global minimum where the piecewise constant and continuous solutions of the problem fall in a local minimum and hence fail to perform the segmentation. Therefore this leads to an unsupervised texture segmentation algorithm based on a Fourier series scheme.

\section{Acknowledgment}

The authors would like to thank the anonymous reviewers for their interesting and constructive suggestions, which helped to improve the presentation of this paper.

\section{References}

1 Kass, M., Witkin, A., and Terzopoulos, D.: 'Snakes: active contour models', Int. J. Comput. Vis., 1987, 1, pp. 321-331

2 Mumford, D., and Shah, J.: 'Optimal approximations by piecewise smooth functions and associated variational problems', Commun. Pure Appl. Math., 1989, 42, (4), pp. 577-688

3 Tsai, A., Yezzi, A., and Willsky, A.S.: 'Curve evolution implementation of the Mumford-Shah functional for image segmentation, denoising, interpolation and magnification', IEEE Trans. Image Process., 2001, 10, (8), pp. 1169-1186

4 Chan, T.F., and Vese, L.A.: 'Active contours without edges', IEEE Trans. Image Process., 2001, 10, (2), pp. 266-277

5 Hintermuller, M., and Ring, W.: 'An inexact-CG-type active contour approach for the minimization of the Mumford-Shah Functional', J. Math. Imaging Vis., 2004, 20, pp. 19-42,

6 Dervieux, A., and Thomasset, F.: 'A finite element method for the simulation of Rayleigh-Taylor instability', in 'Approximation methods for Navier-Stokes problems, lecture notes in mathematics' (Springer, 1979), vol. 771, pp. 145-158

7 Dervieux, A., and Thomasset, F.: 'Multifluid incompressible flows by a finite element method', Lect. Notes Phys., 1981, 11, pp. 158-163

8 Osher, S., and Sethian, J.: 'Fronts propagating with curvature-dependent speed: algorithms based on Hamilton-Jacobi formulations', J. Comput. Phys., 1988, 79, pp. 12-49

9 Sethian, J.A.: 'Levet set methods: evolving interfaces in geometry, fluid mechanics, computer vision and material science' (Cambridge University Press, 1996)

10 Caselles, V., Kimmel, R., and Sapiro, G.: 'Geodesic active contours'. Proc. 5th Int. Conf. on Computer Vision, IEEE Computer Society Press, 1995, pp. 694-699

11 Caselles, V., Kimmel, R., and Saprio, G.: 'Geodesic active contours', Int. J. Comput. Vis., 1997, 22, (1), pp. 61-79

12 Sapiro, G.: 'Geometric partial differential equations and image analysis' (Cambridge University Press, 2001)

13 Kichenassamy, S., Kumar, A., Olver, P., Tannenbaum, A., and Yezzi, A.: 'Gradient flows and geometric active contour models'. Fifth Int. Conf. on Computer Vision (ICCV'95), 1995, pp. 810-815

14 Yezzi, A., Kichenassamy, S., Kumar, A., Olver, P., and Tannenbaum, A.: 'A geometric snake model for segmentation of medical imagery', IEEE Trans. Med. Imaging, 1997, 16, (12), pp. 199-209

15 Kichenassamy, S., Kumar, A., Olver, P., Tannenbaum, A., and Yezzi, A.: 'Conformal curvature flows: from phase transitions to active vision', Arch. Ration. Mech. and Anal., 1996, 134, (3), pp. 275-301

16 Aubert, G., and Kornprobst, P.: 'Mathematical problems in image processing: partial differential equations and calculus of variations' (Springer-Verlag, New York, 2002) 
17 Morel, J.L., and Solimini, S.: 'Variational methods in image segmentation' (Birkhauser, Boston, 1995)

18 Mumford, D., and Shah, J.: 'Boundary detection by minimizing functionals'. Proc. Int. Conf. on Computer Vision and Pattern Recognition, 1985 , pp. $22-26$

19 Vese, L.A., and Chan, T.F.: 'A multiphase level set framework for image segmentation using the Mumford and Shah model', Int. J. Comput. Vis., 2002, 50, (3), pp. 271-293

20 Chan, T.F., Sandberg, B.Y., and Vese, L.A.: 'Active contours without edges for vector-valued images', J. Vis. Commun. Image Represent., 2000, 11, pp. 130-141

21 Chan, T.F., and Vese, L.A.: 'Levels et algorithm for minimising the Mumford-Shah functional in image processing'. Proc. IEEE Workshop on Variational and Level Set Methods in Computer Vision, 2001, pp. 161-168
22 Vese, L.A.: 'Multiphase object detection and image segmentation', Osher, S., and Paragios, N. (Eds.): 'Geometrical level set methods in imaging, vision, and graphics' (Springer Verlag, 2003), pp. $175-194$

23 Gelfand, I.M., and Fomin, S.V.: 'Calculus of variations' (Prentice-Hall Inc., New Jersey, 1963)

24 Mahmoodi, S., and Sharif, B.S.: 'Signal segmentation and denoising algorithm based on energy optimization', Signal Process., 2005, 85, (9), pp. 1845-1851

25 Mahmoodi, S., and Sharif, B.S.: 'Noise reduction, smoothing and time interval segmentation of noisy signals using an energy optimisation method', IEE Proc., Vis. Image Signal Process., 2006, 153, (2), pp. $101-108$

26 Kreyszig, E.: 'Advanced engineering mathematics' (John Wiley, 1999) 